\title{
ELECTRONIC RHEOTOME AND ITS APPLICATION FOR STIMULATION OF THE HUMAN EYE
}

\author{
TOSISADA MITA, ENKICHI FUJIMAKI AND CHUITI SATO* \\ Physiological Laboratory, Iwate Medical College, Morioka, Japan
}

Electric stimulation of the human eye by the sinusoidal alternating currents (A.C.) or the periodic rectangular pulses was studied recently by Rohracher (11), Schwarz (12), Pollock and Mayer (10), Barnett (2), Motokawa and Iwama (9), Mita, Abe and Byonshik (7), Abe (1), Tukahara and Abe (13), Meyer-Schwickerath and Magun (6), Bouman, T. Doesschate and Van der Velden (3), Gebhard (5), Motokawa and Ebe (8).

In their experiments the stimuli were given generally in such a manner that the intensity of the current was progressively increased, till the subject perceived the flickering phosphenes. But their methods are considered to be not satisfactory enough to determine the electric threshold of the eye, because the electric threshold seems to increase rapidly, when the alternating currents flow through the eyes, as pointed out by Pollock and Mayer as an adaptationlike phenomenon. In order to avoid this undesirable effect of the gradually increasing stimuli, Abe attempted in his 2nd experiment to determine the thresholds for a series of the periodic rectangular pulses by using an ordinary spring rheotome. But his method also seems to be not suitable for measurements by the sinusoidal alternating currents, because the electric threshold determined by the sinusoidal alternating currents will depend upon the phase of the current at the beginning and the termination of stimulation, as expected from Copée's studies (4) on nerves and muscles.

It may be, therefore, the most important condition for electrostimulation of the eye by the sinusoidal alternating current, that the alternating current for stimulation starts at the phase of $0^{\circ}$ and ends at the phase of $180^{\circ}$ or $360^{\circ}$ irrespectively of cycles, strength and duration of stimulation.

Considering these requirements, we made an electronic device sufficiently suitable for the purpose stated above and named the apparatus "electronic rheotome." As we are convinced of the wide application of the electronic rheotome not only in the case of stimulation of the eye but also of other tissues, its details and some interesting results obtained by its application for stimulation of: the eye will be given in this communication.

\section{THE PRINCIPLE OF THE ELECTRONIC RHEOTOME}

\section{(A) The 1st type of the electronic rheotome}

Fig. 1 shows the circuit diagram of the electronic rheotome. An oscillator generates the stationary sinusoidal alternating currents. A portion of the cur-

Received for publication October 2, 1953.

* 三田俊定 藤巻延吉 佐藤忠一 


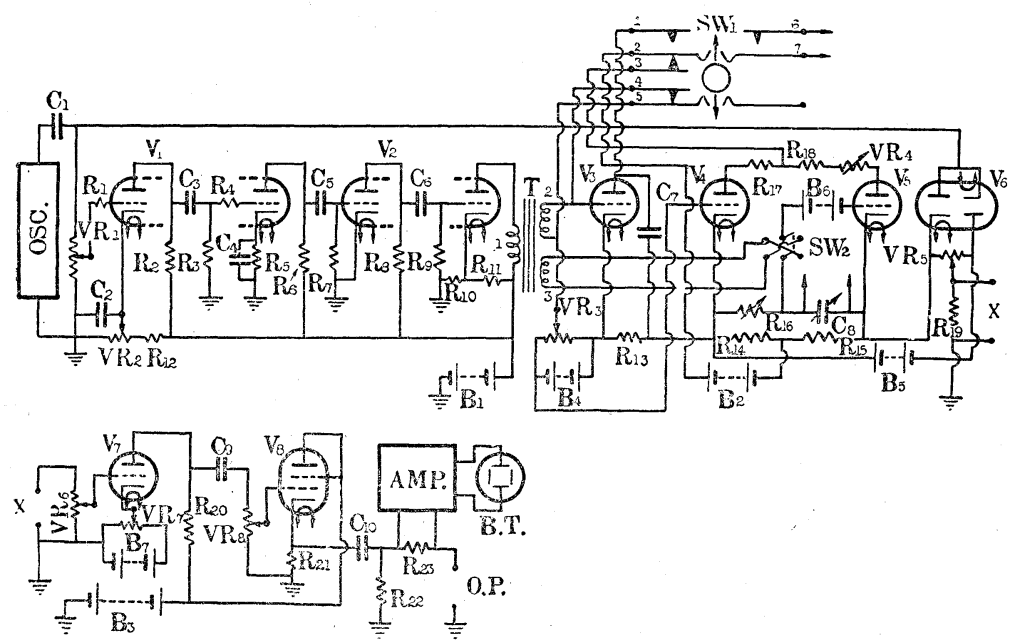

FIG. 1. Circuit diagram of the electronic rheotome of the 1st type.

\begin{tabular}{|c|c|c|}
\hline$R_{1}, R_{3}, R_{4}, R_{22 .} \cdots 500 \mathrm{~K} \Omega$ & $V R_{1}, \ldots \cdots \cdots \cdots 100 \mathrm{~K} \Omega$ & $B_{1}, B_{2} . \cdots \cdots \cdots \cdots 50 \mathrm{~V}$ \\
\hline$R_{2}, R_{6}, R_{8}, R_{20} . \cdots 100 \mathrm{~K} \Omega$ & $V R_{2}, V R_{3}, V R_{5}, V R_{7}$ & $B_{3 .} \quad \cdots \ldots \ldots \ldots \quad 150 \mathrm{~V}$ \\
\hline$R_{5}, R_{12} \ldots \ldots \ldots \ldots 5 \mathrm{~K} \Omega$ & $\cdots \cdots \cdots \cdots \cdots 1 \mathrm{~K} \Omega$ & $B_{4 .} \ldots \ldots \ldots \ldots \ldots$ \\
\hline$R_{7}, R_{9} \ldots \ldots \ldots 250 \mathrm{~K} \Omega$ & $V R_{4} \cdot \cdots \cdots \cdots \cdots+500 \Omega$ & .12 \\
\hline$R_{10}, R_{13 .} \cdots \ldots \ldots 1 \mathrm{~K} \Omega$ & $V R_{6}, V R_{8} . \cdots \cdots 500 \mathrm{~K} \Omega$ & $B_{6}, \ldots \ldots \ldots$ \\
\hline$R_{11}, R_{19} \ldots \ldots \ldots 10 \mathrm{~K} \Omega$ & $C_{1} \ldots \ldots \cdots \cdots \cdots 32 \mu \mathrm{F}$ & $V_{1}, V_{2}, \cdots \cdots \epsilon$ \\
\hline$R_{14}, R_{15} \cdot \cdots \cdots \cdots 240 \Omega$ & $C_{2}, C_{4} . \cdots \cdots \cdots 100 \mu \mathrm{F}$ & $V_{3}, V_{4}, V_{5}$. \\
\hline$R_{16} \cdot \cdots \cdots \cdots, \cdots, 3 \mathrm{M} \Omega$ & $\cdots 4 \mu \mathrm{F}$ & $\cdots 6 \mathrm{H} 6$ \\
\hline$\ldots \ldots \cdots 455 \Omega$ & $\begin{array}{ll}0.5 \mu \mathrm{F} \\
.0 .01 \mu \mathrm{F}\end{array}$ & $V_{7}$. \\
\hline$\cdots 210 \Omega$ & $.0 .1-2 \mu \mathrm{F}$ & $\ldots \ldots \ldots \ldots+807$ \\
\hline$R_{21} \cdot \cdots \cdots \cdots \cdots \quad \cdots 2 \mathrm{~K} \Omega$ & $C_{9} \cdot \cdots \cdots \cdots \cdots \cdots, \mu \mathrm{F}$ & OSC. $\cdots$ C-R Oscillator \\
\hline$R_{23 .} \cdots \cdots \cdots \cdots \cdots \cdots$ & $C_{10} \cdot \cdots \cdots \cdots \cdots \cdot 64 \mu \mathrm{F}$ & B.T. $\cdots \cdots \cdots$ Braun tube \\
\hline
\end{tabular}

rents is introduced to the 4 stage amplifier $\left(V_{1}, V_{2}\right.$, and transformer $T$ ) and is transformed to the periodic spike pulses discharging every phase of $0^{\circ}$ and $180^{\circ}$. When the lever of a switch $S W_{1}$ is located in the middle position, as represented in fig. 1, the periodic pulses induced by the secondary coil 2 of $T$ are shunted by the contact between 4 and 5 of $S W_{1}$.

To make ready for stimulation, the lever of $S W_{1}$ is shifted upward and the condenser $C_{7}$ is charged by battery $B_{2}$. For starting the stimulation, the lever of $S W_{1}$ is lowered downward across the middle position, so as to open the contact between 4 and 5 of $S W_{1}$. Then, the grid bias of a thyratron $V_{3}$ is reduced by the first pulse coming at $0^{\circ}$ phase of A.C. from the secondary coil 2 of $T$, so that its cathode current flows through $R_{13}$ with the discharge of condenser $C_{7}$. At this moment, by the voltage drop induced in $R_{13}$ the grid bias of a thyratron $V_{4}$ is lowered to a point where the plate current of $V_{4}$ can flow. In flowing through $R_{14}$, the cathode current of $V_{4}$ causes the voltage drop in $V R_{5}$ to decrease to nil instantly. As the conductivity of a twin diode $V_{6}$ is controlled by the voltage drop in $V R_{5}$, with the extinction of this voltage drop, the current flows from the oscillator through $V_{6}, V R_{5}$ and $R_{19}$ to ground.

In this way, the flow of the sinusoidal alternating currents through $R_{19}$ can 
be started by a pulse at the phase of $0^{\circ}$.

Meanwhile, when the cathode current of $V_{4}$ flows, the condenser $C_{8}$ begins: to be charged gradually through variable resistor $R_{16}$, so that the grid bias of a thyratron $V_{5}$ decreases gradually too. As the grid bias of $V_{5}$ is superposed with the periodic pulses induced by the secondary coil 3 of $T$, the plate current of $V_{5}$ begins to flow by any pulse as soon as the value of the bias of $V_{5}$ is reduced to a certain level. In flowing through $R_{15}$ the cathode current of $V_{5}$ has an effect of cancelling the voltage drop in $R_{14}$ caused by the cathode current: of $V_{4}$, that is, this flow recovers the voltage drop in $V R_{5}$ again, so as to block the flow of the alternating currents through $V_{6}, V R_{5}$ and $R_{19}$.

Thus, the sinusoidal alternating current flowing through $R_{19}$ can be stopped. by a pulse at the phase of $180^{\circ}$ or $360^{\circ}$. After the alternating current in $R_{19}$ is amplified by $V_{7}$, the output current is taken through the cathode follower $\left(V_{8}\right)$. The wave shape of the output is inspected always on the oscilloscope screen (B.T.). If the beginning and the termination of the currents are observed to be not coincident with the phase of $0^{\circ}$ and $180^{\circ}$ or $360^{\circ}$ respectively, they can be adjusted correctly by regulating the potentiometer $V R_{1}$ or $V R_{2}$. The duration of a stimulus, which, of course, is equal to any multiples of a half period of a cycle, is controlled by $R_{16}$ and $C_{8}$. A switch $S W_{2}$ controls whether the phase at the termination of the current is $180^{\circ}$ or $360^{\circ}$. The output amplitude of the current can be regulated by the potentiometers $V R_{6}$ and $V R_{8}$ precisely. The amplitude at maximum is approximately $4 \mathrm{~V}$ in the case of the load impedance of $1 \mathrm{~K} \Omega$. As the frequency range of our oscillator is from 2 to 1,000 cycles per second (cps.), such factors of a stimulus as the intensity, the frequency and the duration (multiples of a half cycle) can be chosen at will by proper manipulation of the apparatus. When a trial of stimulation ends, the level of $\mathrm{S} W_{1}$ must be shifted upward for opening the plate circuits of $V_{4}$ and $V_{5}$ and for the discharge of $C_{8}$.

\section{(B) The 2nd type of the electronic rheotome}

Although we were at first, rather satisfied with the apparatus stated above, we improved the apparatus in certain respects for further studies.

The one unsatisfactory point is that the coupling condensers $C_{1}$, and $C_{10}$ in fig. 1 need very large capacities in cases of very low frequencies below $10 \mathrm{cps}$. The other is that the output current shows a small amount of transient phenomena (i.e. a few per cent decrease in amplitude of the first half cycle and increase in amplitude of the second half cycle etc.), an inevitable consequence of the condenser coupling. In order to eliminate these inadequacies, we adopted. the direct coupling circuit instead of the condenser coupling. In addition to this improvement, we designed an additional plan for obtaining waves of different forms such as rectangular, rectified anodal and cathodal waves. Fig. 2 shows the circuit diagram of the 2 nd improved type. As the main circuit is similar to the first type mentioned above, only the improved part will be stated here. Both inputs $\left(I P_{1}, I P_{2}\right)$ have to be connected to an ordinary oscillator (omitted from fig. 2). $\quad R_{1}$ and $R_{2}$ are used not only for the regulation of intensity and phase shifting of the pulses, but also for controlling the ratio of the positive interval to the negative interval within a cycle of the rectangular waves. $R_{3}$, 


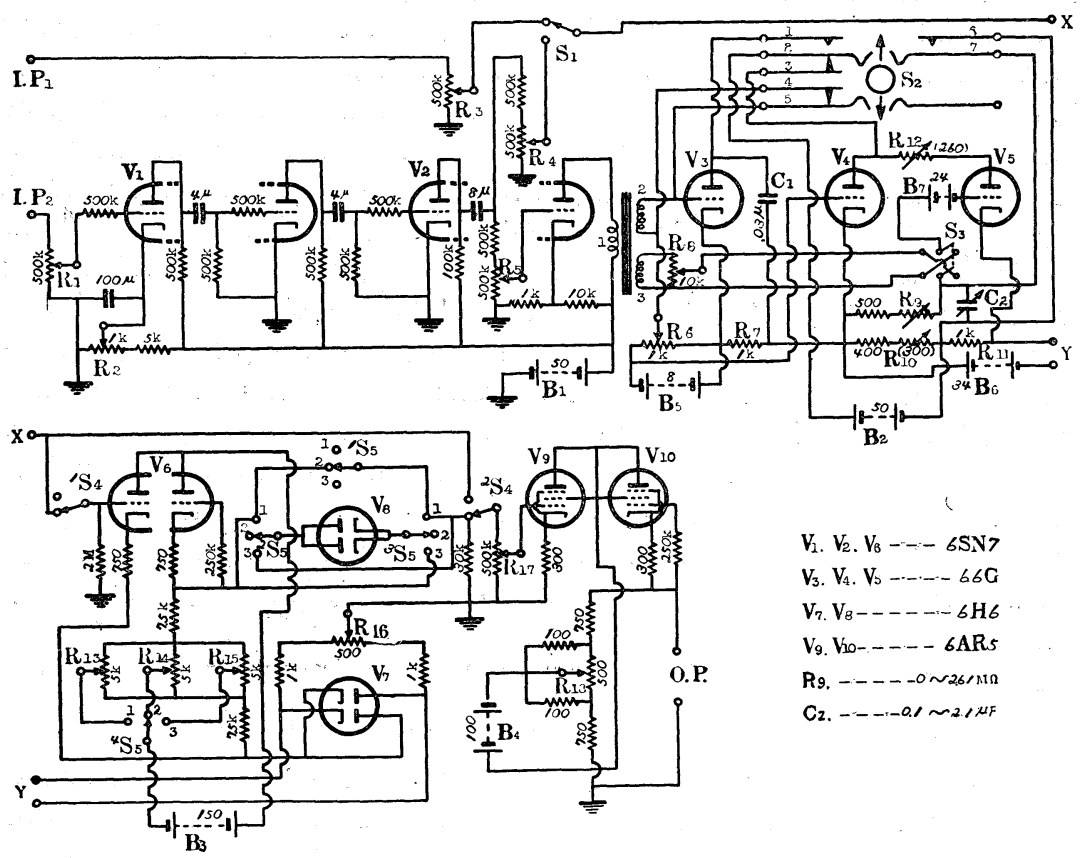

FIG. 2. Circuit diagram of the electronic rheotome of the 2nd type.
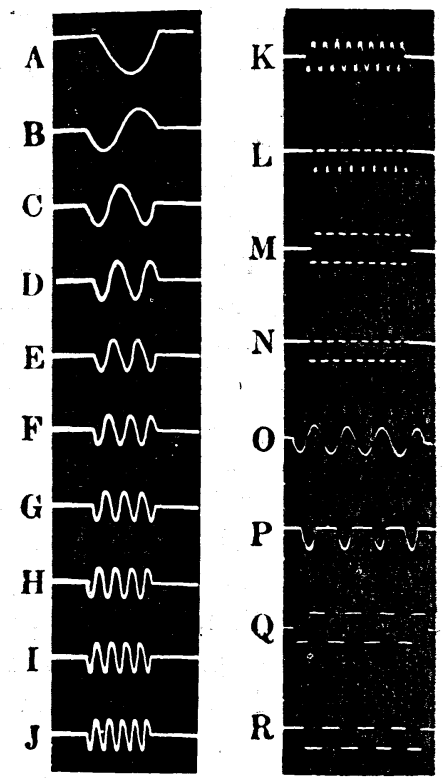

FJG. 3. Wave-shapes from output of the electronic rheotome of the 2nd type.

$A$ : a half cycle of $3 \mathrm{cps}$. sine wave, $B$ : a cycle of 6 cps. sine wave, $C: 1.5$ cycles of $9 \mathrm{cps}$. sine wave, $D: 2$ cycles of $12 \mathrm{cps}$. sine wave, $E: 2.5$ cycles of 15 cps. sine wave, $F: 3$ cycles of 18 cps. sine wave, $G$ : 3.5 cycles of $21 \mathrm{cps}$. sine wave, $H: 4$ cycles of $24 \mathrm{cps}$. sine wave, $I: 4.5$ cycles of $27 \mathrm{cps}$. sine wave, $J: 5$ cycles of $30 \mathrm{cps}$. sine wave, $K: 9$ cycles of $40 \mathrm{cps}$. sine wave, $L: 9$ cycles of rectified sine wave of $40 \mathrm{cps}$., $M$ : 9 cycles of symmetrical square wave of $40 \mathrm{cps}$., $N: 9$ cycles of cathodal square wave of $40 \mathrm{cps}$., $O: 4$ cycles of sine wave of $60 \mathrm{cps}$., $P: 4$ cycles of rectified sine wave of $60 \mathrm{cps}$., $Q: 4$ cycles of symmetrical square wave of 60 cps., $R: 4$ cycles of cathodal square wave of $60 \mathrm{cps}$. 
$R_{4}$ and $R_{5}$ are the attenuators for controlling amplitude of sine waves, rectangular waves and spike pulses respectively. $R_{17}$ is a two-dial attenuator for precise control of the output current. All $R_{13}, R_{14}, R_{15}, R_{16}$ and $R_{18}$ are semifixed potentiometers. At first, $R_{18}$ is adjusted in such a manner that no potential difference remains between the output terminals when the attenuator $R_{17}$ is shunted to ground, whatever the output impedance may be. Then, $R_{14}$ is adjusted so as no current flows through $R_{17}$, when $R_{3}$ is shunted to ground and circuit $Y$ is opened. Finally, still shunting $R_{3}$ to ground, $R_{16}$ is adjusted so as no current flows through $R_{17}$, even when the voltage drop in $R_{16}$ varies with flows of the cathode currents of $V_{4}$ and $V_{5}$. By these adjustments of $R_{18}, R_{14}$ and $R_{16}$, the component of the direct current in output can be reduced far below the noise level of the output current. A switch $S_{5}\left({ }^{1} S_{5},{ }^{2} S_{5},{ }^{3} S_{5},{ }^{4} S_{5}\right)$ is employed for rectification of the output current. In this case $R_{13}$ and $R_{15}$ must be adjusted like in the case of $R_{14}$. A switch $S_{4}\left({ }^{1} S_{4},{ }^{2} S_{4}\right)$ is used for obtaining the continuous flow of the output current. Fig. 3 shows several examples of the output current photographed. The output amplitude (peak voltage) at maximum is about $6.4 \mathrm{~V}$ when the load impedance is $2 \mathrm{~K} \Omega$.

\section{2. electrostimulation of the human Eye}

By means of the electronic rheotome, we examined the dependence of the electric threshold of the eye upon the frequency of A.C. and upon its duration corresponding to different multiples of a half cycle. The measurements were made on two adaptation levels of the eye to dim and bright illumination. Two electrodes, shaped like a shallow cap made of rubber, in whose inner cavity a silver circular disc of $2 \mathrm{~cm}$. diameter is fixed, were fastened watertightly to the forehead and the temple with collodion. Saturated saline, poured into the cavity, is used for contact of the electrode to the skin. The area of the contact surface is. about $7 \mathrm{~cm}^{2}$.

Tables 1 and 2 show two series of the data obtained from the light-adapted and dark-adapted eyes respectively. As shown in tables 1 and 2 , the dependence of the electric threshold on the duration (number of cycles) at a constant frequency is quite different between both ranges of frequency above and below a definite frequency (this critical frequency is about $50-60 \mathrm{cps}$.). The circumstance can be illustrated most clearly by graphical representation. Data of table 1 were plotted in fig. 4. Fig. 4 shows that each curve of 80, 100, 120 and $150 \mathrm{cps}$. has a peculiar oscillation of the threshold value, but that each curve of 20,50 and $60 \mathrm{cps}$. has no oscillation. Thus, the threshold values for a higher frequency than $60 \mathrm{cps}$. differ strikingly according to whether the phase in the termination of the current is $180^{\circ}$ or $360^{\circ}$. The oscillatory characteristics of the curves are enhanced remarkably at the first few cycles, and the higher the frequency is, the larger becomes the amplitude of the oscillation. The critical frequency, where the oscillation occurs, was not determined precisely in the present experiment. But it seems to be at the vicinity of $50-60 \mathrm{cps}$. and to depend on the adaptation of the eye.

On the other hand, the value of the threshold for a frequency below the critical frequency decreases steadily, corresponding to the increase in duration 
TABLE 1. Electric Threshold Values in MA of the Eye Light-adapted to 2,500 Luxes.

Measured by the Electronic Rheotome of the 1st Type.

Subject: E. Fujimaki

\begin{tabular}{l|l|l|l|l|l|l|l|l|l}
\hline \hline \multirow{2}{*}{$\begin{array}{c}\text { Duration } \\
\text { (number } \\
\text { of cycle }\end{array}$} & \multicolumn{10}{c}{ Frequency } \\
\cline { 2 - 9 } & 10 & 20 & 30 & 50 & 60 & 80 & 100 & 120 & 150 \\
\hline 0.5 & 0.063 & 0.107 & 0.186 & 0.239 & 0.32 & 0.41 & 0.49 & 0.60 & 0.85 \\
1 & 0.052 & 0.077 & 0.101 & 0.192 & 0.27 & 0.37 & 0.63 & 0.78 & 1.21 \\
1.5 & 0.052 & 0.060 & 0.093 & 0.192 & 0.29 & 0.50 & 0.62 & 0.85 & 0.97 \\
2 & 0.052 & 0.047 & 0.086 & 0.192 & 0.30 & 0.40 & 0.56 & 0.70 & 0.99 \\
2.5 & 0.052 & 0.045 & 0.086 & 0.192 & 0.32 & 0.48 & 0.71 & 0.77 & 1.21 \\
3 & 0.052 & 0.045 & 0.082 & 0.192 & 0.31 & 0.43 & 0.54 & 0.76 & 1.05 \\
3.5 & 0.052 & 0.031 & 0.082 & 0.172 & 0.31 & 0.43 & 0.74 & 0.88 & 1.22 \\
4 & 0.052 & 0.031 & 0.078 & 0.176 & 0.29 & 0.43 & 0.58 & 0.83 & 1.05 \\
4.5 & 0.052 & 0.031 & 0.078 & 0.172 & 0.29 & 0.41 & 0.68 & 1.01 & 1.18 \\
5 & 0.051 & 0.030 & 0.075 & 0.172 & 0.27 & 0.42 & 0.77 & 0.99 & 1.08 \\
5.5 & 0.051 & 0.030 & 0.075 & 0.164 & 0.28 & 0.42 & 0.75 & 1.16 & 1.38 \\
$\mathrm{M}$ & 0.035 & 0.030 & 0.075 & 0.164 & 0.26 & 0.45 & 0.89 & 0.93 & 1.40 \\
\hline
\end{tabular}

TABLE 2. Electric Threshold Values in MA of the Dark-adapted Eye. Measured by the Electronic Rheotome of the 1st Type.

Subject: E. Fujimaki

\begin{tabular}{|c|c|c|c|c|c|c|c|c|c|}
\hline \multirow{2}{*}{$\begin{array}{c}\text { Duration } \\
\left(\begin{array}{c}\text { number } \\
\text { of cycle }\end{array}\right)\end{array}$} & \multicolumn{9}{|c|}{ Frequency (cps.) } \\
\hline & 10 & 20 & 30 & 50 & 60 & 80 & 100 & 120 & 150 \\
\hline 0.5 & 0.159 & 0.160 & 0.136 & 0.244 & 0.28 & 0.58 & 0.81 & 0.52 & 1.14 \\
\hline 1 & 0.102 & 0.103 & 0.082 & 0.187 & 0.26 & 0.54 & 0.86 & 0.72 & 1.39 \\
\hline 1.5 & 0.079 & 0.086 & 0.074 & 0.197 & 0.30 & 0.74 & 0.89 & 0.69 & 1.08 \\
\hline 2 & 0.072 & 0.086 & 0.066 & 0.220 & 0.29 & 0.62 & 0.78 & 0.58 & 0.92 \\
\hline 2.5 & 0.077 & 0.074 & 0.062 & 0.232 & 0.28 & 0.59 & 0.96 & 0.91 & 1.20 \\
\hline 3 & 0.065 & 0.077 & 0.058 & 0.203 & 0.28 & 0.63 & 0.85 & 0.65 & 0.95 \\
\hline 3.5 & 0.065 & 0.074 & 0.054 & 0.226 & 0.28 & 0.63 & 0.78 & 0.78 & 1.11 \\
\hline 4 & 0.062 & 0.077 & 0.054 & 0.190 & 0.28 & 0.60 & 0.80 & 0.74 & 0.97 \\
\hline 4.5 & 0.057 & 0.086 & 0.054 & 0.226 & 0.28 & 0.64 & 0.86 & 0.74 & 1.11 \\
\hline 5 & 0.054 & 0.083 & 0.054 & 0.244 & 0.29 & 0.62 & 0.82 & 0.73 & 0.99 \\
\hline 5.5 & 0.057 & 0.083 & 0.054 & 0.232 & 0.28 & 0.62 & 0.82 & 0.72 & 1.08 \\
\hline $\mathbf{M}$ & 0.065 & 0.086 & 0.049 & 0.226 & 0.29 & 0.62 & 0.93 & 0.72 & 1.11 \\
\hline
\end{tabular}

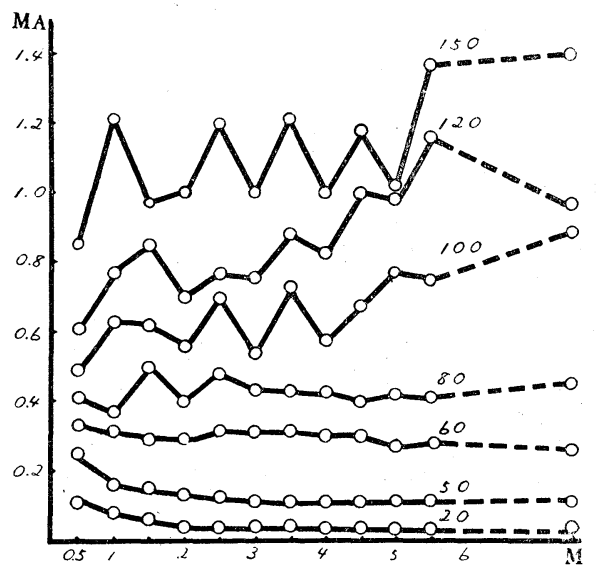

FIG. 4. Strength-duration curves of the light-adapted eye. Ordinate shows the threshold values in MA. Abscissa is the duration counted in number of cycles. Number on the curve is the frequency of A.C. $M$ on abscissa means many cycles (very long duration). 
of stimulation (counted in number of cycles) and reached the lowest value in the duration of 2 to 3 cycles, thereafter remaining almost constant, no matter which phase of $180^{\circ}$ or $360^{\circ}$ may be chosen at the termination of the stimulating current. It was found, therefore, that only a few cycles at the onset of the current cause the summation of stimuli in the case of the lower frequency. This summation seems to become more evident in eyes adapted to dim light, especially in the frequency of $15-20 \mathrm{cps}$.

\section{SUMMARY}

An apparatus named "electronic rheotome" was devised by us for electric stimulation of the eye with sinusoidal alternating currents. The electronic rheotome enables us to start the aiternating currents at $0^{\circ}$ phase and to stop them at $180^{\circ}$ or $360^{\circ}$ phase. Combining the electronic rheotome with an A.C. oscillator, we can settle precisely such factors of a stimulus as intensity, duration, frequency and phase of the beginning and the termination, that the measurements of the electric threshold can be made accurately. The electronic rheotome of the 2nd type, furthermore, can produce currents of different forms such as square, anodal and cathodal waves.

As a first application of the electronic rheotome we examined the dependence of the electric threshold of the eye upon duration and frequency of the sinusoidal alternating currents. It was found that the characteristics of the strength-duration curve at any frequency are quite different between both ranges above and below a definite frequency in the vicinity of 50 to $60 \mathrm{cps}$. The threshold values for a frequency above $60 \mathrm{cps}$. differ strikingly according to whether the phase at the termination of the current is $180^{\circ}$ or $360^{\circ}$, while the threshold values for a frequency below $50 \mathrm{cps}$. decrease steadily with the increase in duration of stimulation, till they reach the lowest value in the duration of 2 to 3 cycles, and they thereafter remain almost constant, no matter at which phase of $180^{\circ}$ or $360^{\circ}$ the stimulating currents end. That is, a few cycles at the onset of the stimulating currents cause the summation of stimuli. This summation of stimuli takes place most remarkably in the neighbourhood of $20 \mathrm{cps}$.

\section{REFERENCES}

1. ABE, Z. Tohoku J. exp. Med. 54: 37, 1951.

2. Barnett, A. Am. J. Physiol. 133: 205, 1941.

3. Bouman, M. A., T. Doesschate, J. And VAn DeR Velden, H. A. Documenta Ophthal. 5: 151, 1951.

4. Coppée, G. Cold Spr. Harb. Sympos. quant. Biol. 4: 150, 1936.

5. Gebhard, J. W. J. exp. Psychol. 44: 132, 1952.

6. MEYER-SCHWICKERATH, G. AND MAGUN, R. v. Graefes Arch. Ophthalmol. $151:$ 693, 1951.

7. MitA, T., ABE, Z. AND Byonshik, T. Tohoku J. exp. Med. 54: 45, 1951.

8. MOTOKAWA, K. AND EBE, M. Science, 116: 92, 1952.

9. MotoKAWA, K. AND IW AMA, K. Tohoku J. exp. Med. 53: 201, 1950.

10. Pollock, L. T. ANd Mayer, L. L. Am. J. Physiol. 122: 57, 1938.

11. ROHRACHER, H. Z. Sinnesphysiol. $66: 164,1935$.

12. SCHWARz, F. Z. Sinnesphysiol. 67: 227; 68: 92; 69: 1; 69: 158, 1936-1941. Pflüg. Arch. ges. Physiol. 249 : 76, 1947.

13. TukaharA, S. AND ABE, Z. Tohoku J. exp. Med. 54: 189, 1951. 